\title{
Longitudinal and transverse parton momentum distributions for pion and nucleon within relativistic constituent quark models
}

\author{
T. Frederico ${ }^{1}$, E.Pace ${ }^{2, a}$, B. Pasquini ${ }^{3}$, and G. Salmè ${ }^{4}$ \\ 1 Dep. de Física, Instituto Tecnológico de Aeronáutica, 12.228-900 São José dos Campos, São Paulo, Brazil \\ 2 Dipartimento di Fisica, Università degli Studi di Roma "Tor Vergata" and Istituto Nazionale di Fisica Nucleare, Sezione \\ Tor Vergata, Via della Ricerca Scientifica 1, 00133 Roma, Italy \\ 3 Dipartimento di Fisica Nucleare e Teorica, Università degli Studi di Pavia and Istituto Nazionale di Fisica Nucleare, \\ Sezione di Pavia, Pavia, Italy \\ 4 Istituto Nazionale di Fisica Nucleare, Sezione di Roma, P.le A. Moro 2, 00185 Roma, Italy
}

\begin{abstract}
Longitudinal and transverse quark momentum distributions for the pion and the nucleon are calculated from quark-hadron vertexes obtained by an investigation of electromagnetic form factors within relativistic constituent quark models. The no-helicity flip generalized parton distributions for the pion are evaluated within three different models. The relevance for the pion of the one-gluon-exchange dominance at short range for the behavior of the form factor at large momentum transfer and of the parton distributions at the end points is shown.
\end{abstract}

\section{Introduction}

Extensive theoretical and experimental research programs are being pursued to gain information on generalized parton distributions (GPD's), which give access to a detailed description of hadron structure (see.e.g., Refs. [1-3]).

Our strategy to this end is to determine the hadron vertex function from an investigation of hadron electromagnetic (em) form factors (ff) in the spacelike (SL) region within relativistic constituent quark models, and then to use the obtained vertex function to evaluate the GPD's.

In this contribution we shortly review :

- our results for the unpolarized GPD's of the pion within covariant and light-front (LF) models [4];

- our preliminary results for the unpolarized longitudinal and transverse parton momentum distributions (TMD) in the nucleon within a LF framework [5].

Three pion models are used: i) an analytic covariant model with a bare photon vertex; ii) a Mandelstam inspired LF model with a dressed photon vertex; iii) a LF Hamiltonian dynamics (LFHD) model with a bare photon vertex.

In order to study the GPD's in the valence region as well as in the nonvalence (NV) region, the Fock state decomposition [6] of the pion and of the nucleon state is considered :

$$
\begin{gathered}
|\pi\rangle=\underbrace{|q \bar{q}\rangle}_{\text {valence }}+\underbrace{|q \bar{q} q \bar{q}\rangle+|q \bar{q} g\rangle \ldots \ldots}_{\text {valence }} \\
\mid \text { nucleonvalence }\rangle=\underbrace{|q q q\rangle}_{\text {nonvalence }}+\underbrace{|q q q q \bar{q}\rangle+|q q q g\rangle \ldots . . .}
\end{gathered}
$$

\section{Pion generalized parton distributions}

Isoscalar and isovector pion GPD's in the light-cone gauge are

$H_{\pi}^{0}(x, \xi, t)=\left.\int \frac{d z^{-}}{4 \pi} e^{i x P^{+} z^{-}}\left\langle p^{\prime}\left|\bar{\psi}_{q}\left(-\frac{z}{2}\right) \gamma^{+} \psi_{q}\left(\frac{z}{2}\right)\right| p\right\rangle\right|_{\tilde{z}=0}$

$H_{\pi}^{1}(x, \xi, t)=\left.\int \frac{d z^{-}}{4 \pi} e^{i x P^{+} z^{-}}\left\langle p^{\prime}\left|\bar{\psi}_{q}\left(-\frac{z}{2}\right) \gamma^{+} \tau_{3} \psi_{q}\left(\frac{z}{2}\right)\right| p\right\rangle\right|_{\tilde{z}=0}$

where $\tilde{z} \equiv\left\{z^{+}=z^{0}+z^{3}, \mathbf{z}_{\perp}\right\}$, and $\psi_{q}(z)$ is the quark field isodoublet, while

$$
\begin{gathered}
P=\frac{1}{2}\left(p^{\prime}+p\right) \quad \Delta=p^{\prime}-p \\
x=\frac{k^{+}}{P^{+}} \quad \xi=-\frac{p^{\prime+}-p^{+}}{2 P^{+}} \quad t=\Delta^{2}
\end{gathered}
$$

and $k$ is the average momentum of the active quark, i.e. the one that interacts with the photon (see Fig. 1). The variable

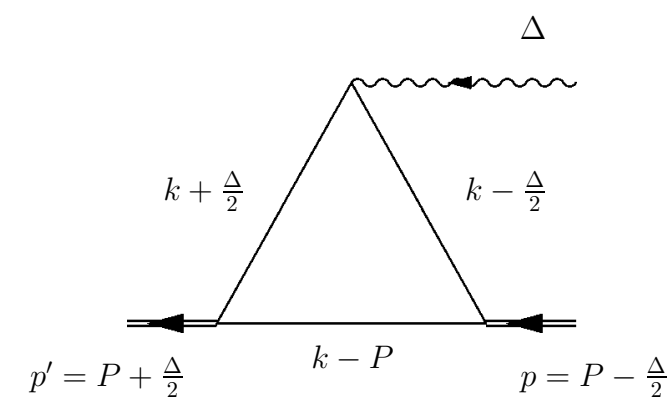

Fig. 1. Diagrammatic representation of the pion GPD, with four momentum definitions (after Ref. [4]). 


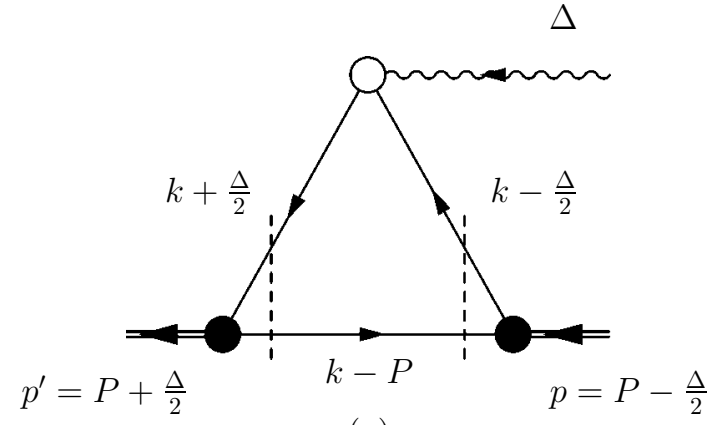

(a)

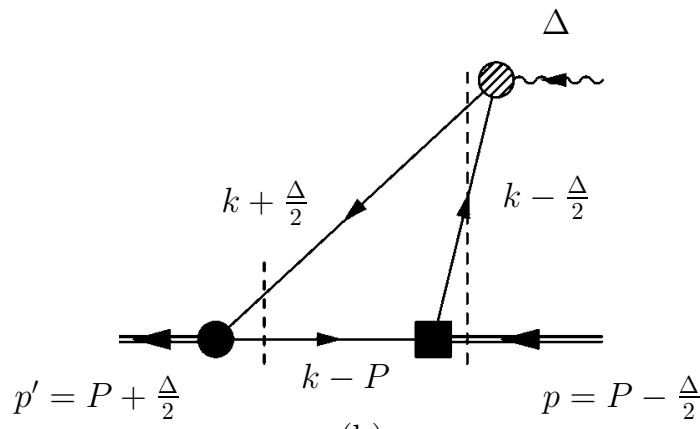

(b)

Fig. 2. LF time-ordered analysis of the pion GPD. Diagram (a): a contribution of the active-quark in the valence region with $1 \geq x \geq|\xi|$. Diagram (b) : a contribution (pair-production process) in the nonvalence region, $|\xi|>x>-|\xi|$ (after Ref. [4]).

$x$ allows one to single out (i) the valence region (DGLAP [7]), for $1 \geq x \geq|\xi|$ and $-|\xi| \geq x \geq-1$, diagonal in the Fock space; and (ii) the nonvalence region (ERBL $[8,9]$ ), for $|\xi|>x>-|\xi|$, non diagonal in the Fock space.

\section{Pion models}

\subsection{Analytic covariant pion model with symmetric regulators}

We use a pion Bethe-Salpeter amplitude (BSA) suggested by an effective Lagrangian [10]

$\Psi(k-P, p)=-\frac{m}{f_{\pi}} S(k-\Delta / 2) \gamma^{5} \Lambda(k-P, p) S(k-P)(6)$

where $m=220 \mathrm{MeV}$ is quark mass and $f_{\pi}=92.4 \mathrm{MeV}$ the pion decay constant. The free quark propagator, $S(k)$, fully includes the instantaneous term, $\gamma^{+} / 2 k^{+}$,

$$
S(k)=\frac{\not k+m}{k^{2}-m^{2}+l \epsilon}=\frac{\not k_{o n}+m}{k^{+}\left(k^{-}-k_{o n}^{-}+\frac{l \epsilon}{k^{+}}\right)}+\frac{\gamma^{+}}{2 k^{+}} .
$$

Two covariant symmetric forms for $\Lambda(k-P, p)$ are used : i) a sum form

$$
\Lambda_{1}=\frac{C_{1}}{\left[(k-\Delta / 2)^{2}-m_{R}^{2}+\imath \epsilon\right]}+\frac{C_{1}}{\left[(P-k)^{2}-m_{R}^{2}+\imath \epsilon\right]}
$$

ii) a product form

$$
\Lambda_{2}=\frac{C_{2}}{\left[(k-\Delta / 2)^{2}-m_{R}^{2}+l \epsilon\right]\left[(P-k)^{2}-m_{R}^{2}+l \epsilon\right]},
$$

where $C_{1}, C_{2}$ are normalization constants. The parameter $m_{R}$ is chosen to fit $f_{\pi}$ in each model.

The $u$-quark GPD in the pion, $H^{u}(x, \xi, t)=H_{\pi}^{I=0}+$ $H_{\pi}^{I=1}$, is given in impulse approximation by

$$
\begin{aligned}
& H^{u}(x, \xi, t)=-\imath N_{c} \mathcal{R} \times \\
& \int \frac{d^{4} k}{2(2 \pi)^{4}} \delta\left(P^{+} x-k^{+}\right) V^{+} \Lambda\left(k-P, p^{\prime}\right) \Lambda(k-P, p)
\end{aligned}
$$

where $N_{c}=3$ is the number of colors, $\mathcal{R}=2 \mathrm{~m}^{2} / f_{\pi}^{2}$, the $\delta$ function imposes the correct support $-|\xi| \leq x \leq 1$ for the active quark and

$$
V^{+}=\operatorname{Tr}\left\{S(k-P) \gamma^{5} S\left(k+\frac{\Delta}{2}\right) \gamma^{+} S\left(k-\frac{\Delta}{2}\right) \gamma^{5}\right\}
$$

We adopt a Breit frame with $\Delta^{+}=-\Delta^{-} \geq 0$. Thus

$$
\xi^{2}=\frac{-\Delta^{2}-\left|\Delta_{\perp}\right|^{2}}{-\Delta^{2}+4 m_{\pi}^{2}}
$$

and the whole kinematical range $-1 \leq \xi \leq 1$ can be explored.

\subsection{Mandelstam-inspired pion light-front model}

In Ref. [11] a model for the pion em ff was presented, based on the covariant Mandelstam formula for the current [12], with a microscopic Vector Meson (VM) dominance dressing for the quark-photon vertex. The dynamical inputs were the pion and vector mesons LF wave functions (wf's), eigenstates of the relativistic constituent quark (CQ) mass operator of Ref. [13].

Extending the approach of [11], one finds for $H^{u}$ the expression of Eq. (10), but the bare quark-photon vertex, $\gamma^{+}$, is replaced by the VM dominance (VMD) vertex [11]

$\Gamma^{+}(k, \Delta)=\sqrt{2} \sum_{n, \lambda}\left[\epsilon_{\lambda} \cdot \widehat{V}_{n}\left(k, P_{n}\right)\right] \Lambda_{n}\left(k, P_{n}\right) \frac{\left[\epsilon_{\lambda}^{+}\right]^{*} f_{V n}}{\left(t-M_{n}^{2}\right)}$

where $f_{V n}$ is the decay constant of the n-th VM (calculated within the model $), P_{n}^{\mu} \equiv\left\{M_{n}^{2} / \Delta^{+}, \Delta^{+}, \mathbf{0}_{\perp}\right\}$ the $\mathrm{VM}$ four-momentum with $P_{n}^{2}=M_{n}^{2}, \epsilon_{\lambda}\left(P_{n}\right)$ the VM polarization, and $\widehat{V}_{n}\left(k, P_{n}\right)$ the proper VM Dirac structure.

$\mathrm{Up}$ to 20 isovector mesons are considered to reach convergence even for $t=-10(\mathrm{GeV} / \mathrm{c})^{2}$.

For this model, in the $k^{-}$integration of Eq. (10), only the propagators poles are considered, i.e. the BSA analytic structure is disregarded both i) in the initial and final pion and ii) in the quark-photon vertex.

For the tridimensional reduction of the VM BSA in the valence sector, $0<k^{+}<P_{n}^{+}$, we take the eigenfunction, $\psi_{n}\left(k^{+}, \mathbf{k}_{\perp} ; P_{n}\right)$, of a relativistic square mass operator [13], 
normalized to the probability of the valence Fock state, according to a simple model [11]:

$$
\frac{\left.P_{n}^{+} \Lambda_{n}\left(k, P_{n}\right)\right|_{k^{-}=k_{o n}^{-}}}{\left[M_{n}^{2}-M_{0}^{2}\left(k^{+}, \mathbf{k}_{\perp} ; P_{n}\right)\right]}=\psi_{n}\left(k^{+}, \mathbf{k}_{\perp} ; P_{n}\right),
$$

where $\left.M_{0}^{2}\left(k^{+}, \mathbf{k}_{\perp} ; P_{n}\right)\right]=P_{n}^{+}\left[k_{\text {on }}^{-}+\left(P_{n}-k\right)_{\text {on }}^{-}\right]$is the mass of the free $q \bar{q}$ system.

For the pion in the valence sector ( $\bullet$ in Fig. 2) the eigenfunction of the mass operator of [13] is used. For the NV pion vertex ( $\boldsymbol{\square}$ in Fig. 2 (a)), we assume a constant [14].

All of the parameters of [11] are used, but for the CQ mass $m=200 \mathrm{MeV}$, instead of $m=265 \mathrm{MeV}$. A parameter, $w=-1$, which modulates the relative weights of our two instantaneous contributions is used to fit the pion ff.

For this model we take a particular Breit frame, where $\boldsymbol{\Delta}_{\perp}=0$, and assume $m_{\pi}=0$ (see Ref. [11]). Then $\xi=-1$ and only the NV region contributes.

\subsection{Light-front Hamiltonian dynamics model}

In the LFHD model [15] the Drell-Yan $\Delta^{+}=0$ reference frame is adopted and then the variable $x$ becomes the longitudinal momentum fraction $x_{q}$, since $\xi=0$ for any $t$.

Within the LFHD model the pion LF wave function is

$$
\begin{aligned}
& \Psi_{\pi}\left(x, \kappa_{\perp} ; \lambda_{q}, \lambda_{\bar{q}}\right)=\psi_{\pi}\left(x, \kappa_{\perp}\right) \\
& \quad \times \sum_{\mu_{q}, \mu_{\bar{q}}}\left(\frac{1}{2} \mu_{q} \frac{1}{2} \mu_{\bar{q}} \mid 00\right) D_{\mu_{q} \lambda_{q}}^{1 / 2 *}[R(\kappa)] D_{\mu_{\bar{q}} \lambda_{\bar{q}}}^{1 / 2 *}[R(-\kappa)]
\end{aligned}
$$

where $\lambda_{i}(i=q, \bar{q})$ are the spin projections, $\kappa \equiv\left\{\kappa_{\perp}, \kappa_{z}\right\}$, $\kappa_{z}=M_{0}\left(x, \kappa_{\perp}\right)\left(x-\frac{1}{2}\right)$ and $M_{0}\left(x, \kappa_{\perp}\right)$ is the pion free mass

$$
M_{0}^{2}\left(x, \kappa_{\perp}\right)=\frac{m^{2}+\left|\kappa_{\perp}\right|^{2}}{x(1-x)} .
$$

The matrix elements of the Melosh rotation $R(\kappa)$

$$
D_{\lambda \mu}^{1 / 2}[R(\kappa)]=\langle\lambda|R(\kappa)| \mu\rangle
$$

convert the instant-form spins into LF spins and ensure the rotational covariance.

For the momentum component of the pion wf we use an exponential form

$$
\psi_{\pi}\left(x, \kappa_{\perp}\right)=\frac{\left[2(2 \pi)^{3}\right]^{1 / 2}}{\pi^{3 / 4} \beta^{3 / 2}}\left(\frac{M_{0}\left(x, \kappa_{\perp}\right)}{4 x(1-x)}\right)^{1 / 2} \exp \left(\frac{-\kappa^{2}}{2 \beta^{2}}\right) .
$$

The constituent quark mass $m=250 \mathrm{MeV}$ and the parameter $\beta=319.4 \mathrm{MeV}$ are adjusted to fit $f_{\pi}$ and the pion charge radius.

In this model the pion GPD $H^{u}(x, \xi=0, t)$ in the range $0 \leq x \leq 1$ is given by a diagonal contribution with $n=2$ constituents

$$
H^{u}=\sum_{\left\{\lambda_{i}\right\}} \int \frac{d \kappa_{\perp}}{2(2 \pi)^{3}} \Psi_{\pi}^{*}\left(x, \kappa_{\perp}^{\prime} ;\left\{\lambda_{i}\right\}\right) \Psi_{\pi}\left(x, \kappa_{\perp} ;\left\{\lambda_{i}\right\}\right)
$$

Initial and final transverse components of active quark momenta in the intrinsic frame are related by $\kappa_{\perp}^{\prime}=\kappa_{\perp}+(1-$ $x) \Delta_{\perp}$. Then at large $|t|$, i.e. at large $\left|\Delta_{\perp}\right|, H^{u}(x, \xi=0, t)$ is expected to be non vanishing only for $x \sim 1$.

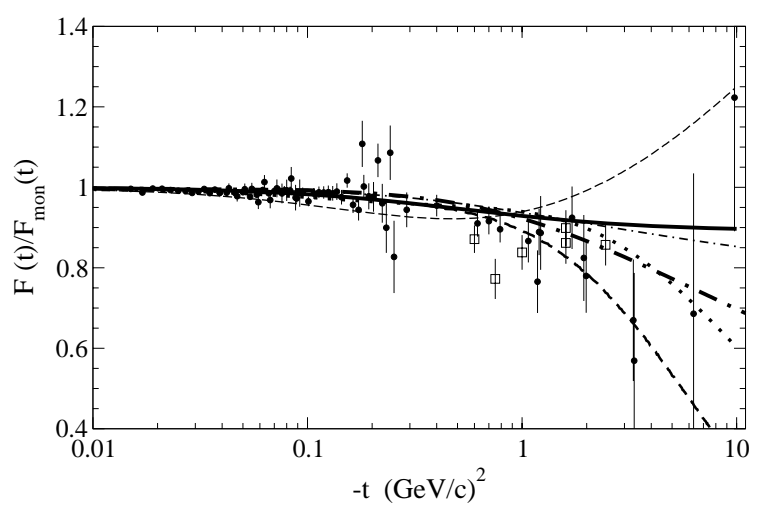

Fig. 3. Pion ff vs $-t$. Thin dashed line: covariant model, with the sum-form BSA. Dotted line: covariant model, with the product-form BSA. Thick solid line and thick dot-dashed line: monopole and faster-than-monopole fit to lattice data [16], arbitrarily extended from -4 to $-10(\mathrm{GeV} / \mathrm{c})^{2}$. Dot-dashed line: $\mathrm{LF}$ Mandelstam-inspired model. Thick dashed line: LFHD model. Experimental data from Refs. [17] (full dots), and [18] (open squares) (adjusted from Ref. [4]).

\subsection{Pion form factor}

The parameters of our three models are adjusted to fit the pion $\mathrm{ff}$, given by the following sum rule

$$
\int_{-1}^{1} d x H_{\pi}^{I=1}(x, \xi, t)=F_{\pi}(t)
$$

Our results for $F_{\pi}(t) / F_{\text {mon }}(t)$ are shown in Fig. 3 and compared with two different fits to lattice data [16] and to experimental data $\left(F_{m o n}(t)=1 /\left(1+|t| / m_{\rho}^{2}\right), m_{\rho}=770 \mathrm{MeV}\right)$.

Let us notice that models which exhibit an asymptotic decay slower than $F_{\text {mon }}(t)$, as the covariant sum-form one does, yield a divergent density at short range.

The valence and NV contributions to the ff were analyzed in [4] for different reference frames.

\section{Pion longitudinal and transverse momentum distributions}

At $\xi=0$ the variable $x$ coincides with the longitudinal momentum fraction $x_{q}$ and for $t=0$ one gets from $H^{u}$ the longitudinal momentum distribution

$$
\begin{gathered}
u(x)=H^{u}(x, 0,0)=2 H_{\pi}^{I=1}(x, 0,0) \\
u(x)=\int d \mathbf{k}_{\perp} f_{1}\left(x, k_{\perp}\right) \quad(x \geq 0),
\end{gathered}
$$

where $f_{1}\left(x, k_{\perp}\right)$ is the TMD. The longitudinal distributions for the covariant and LFHD models are compared in Fig. 4, while the transverse momentum distributions for the two covariant models are shown in Fig. 5.

Notice that the covariant sum-form model is unable to give a vanishing value for $u(x)$ at the end points, while the 


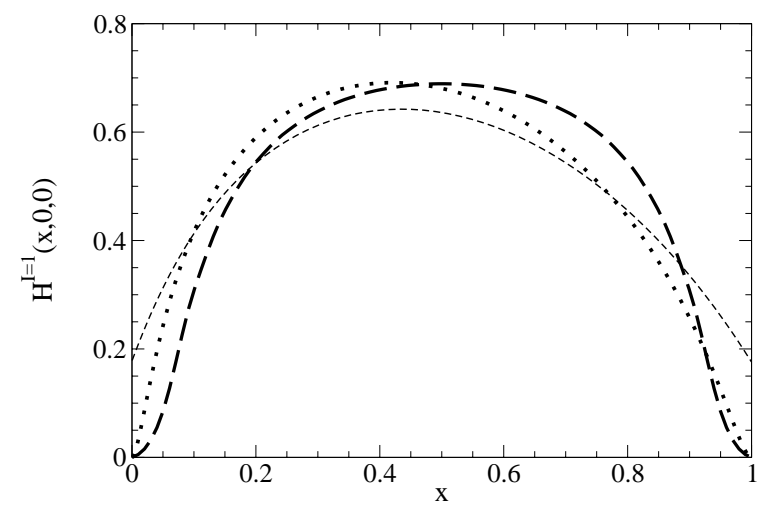

Fig. 4. Thin dashed line: covariant model with the sum-form BSA. Dotted line: covariant model with the product-form BSA. Thick dashed line: LFHD model (after Ref. [4]).

product-form model gives a consistent description both of the $\mathrm{ff}$ tail and of the end-point fall-off of the parton distribution. Indeed, both at $-t \rightarrow \infty$ and at $x \rightarrow 0$ or at $x \rightarrow 1$ the high momentum part of the pion state is probed and the product-form model has a $k_{\perp}^{4}$ fall-off of the BSA, compatible with a BSA kernel dominated by the one-gluon-exchange (OGE) and faster than the $\left|\mathbf{k}_{\perp}\right|$ decay of the sum-form model.

\section{Generalized Parton Distributions}

In Fig. 6, the no-helicity flip isoscalar and isovector GPD's obtained at $|\xi|=1$ (NV region) by the covariant model, for $m_{\pi}=0$ with the product-form BSA, are compared with the results corresponding to the Mandelstam-inspired model, while in Fig. 7 the GPD's obtained by the same covariant model at $\xi=0$ (valence region) are compared with the results of the LFHD model.

Both at $|\xi|=1$ and at $\xi=0$ the covariant productform model is able to show the same general behavior of the other models and for all of the considered models at high $t$ a maximum of GPD's around $x \sim 1$ occurs.

This last fact has a simple kinematical explanation at $\xi=0$, as already shown at the end of Sec. 3.1. Also at $\xi=-1$, where only the $q \bar{q}$ pair production contributes, at high $t$ a maximum of GPD's around $x \sim 1$ is expected. Indeed $\xi=-1$ implies $x=2 k^{+} / \Delta^{+}$, while large $|t|$ values mean large $\Delta^{+}=\Delta_{z} \approx 2 k_{z q}$, and $2 k^{+}=k_{q}^{+}-k_{\bar{q}}^{+} \sim 2 E_{q}=$ $2 \sqrt{m^{2}+\mathbf{k}^{2}}$, since each quark in the pair is almost on its mass shell. Then one has $x \sim E_{q} / k_{z q} \rightarrow 1$.

Eventually, in Fig. 8 the isovector unpolarized GPD at $|\xi|=x, m_{\pi}=0$ for the covariant model with the product form BSA is shown. The GPD at $|\xi|=x$ is relevant because in this case one explores the transition from the valence to the NV region. This kinematical regime should be relevant to study single spin asymmetry [2]. Again, as $-t \rightarrow \infty$, the maximum of GPD moves from $x \sim 0.5$ towards $x=1$.

Since the covariant product-form model is able to reproduce the GPD's evaluated by the Mandelstam-inspired model at $|\xi|=1$ and by the LFHD model at $\xi=0$, one
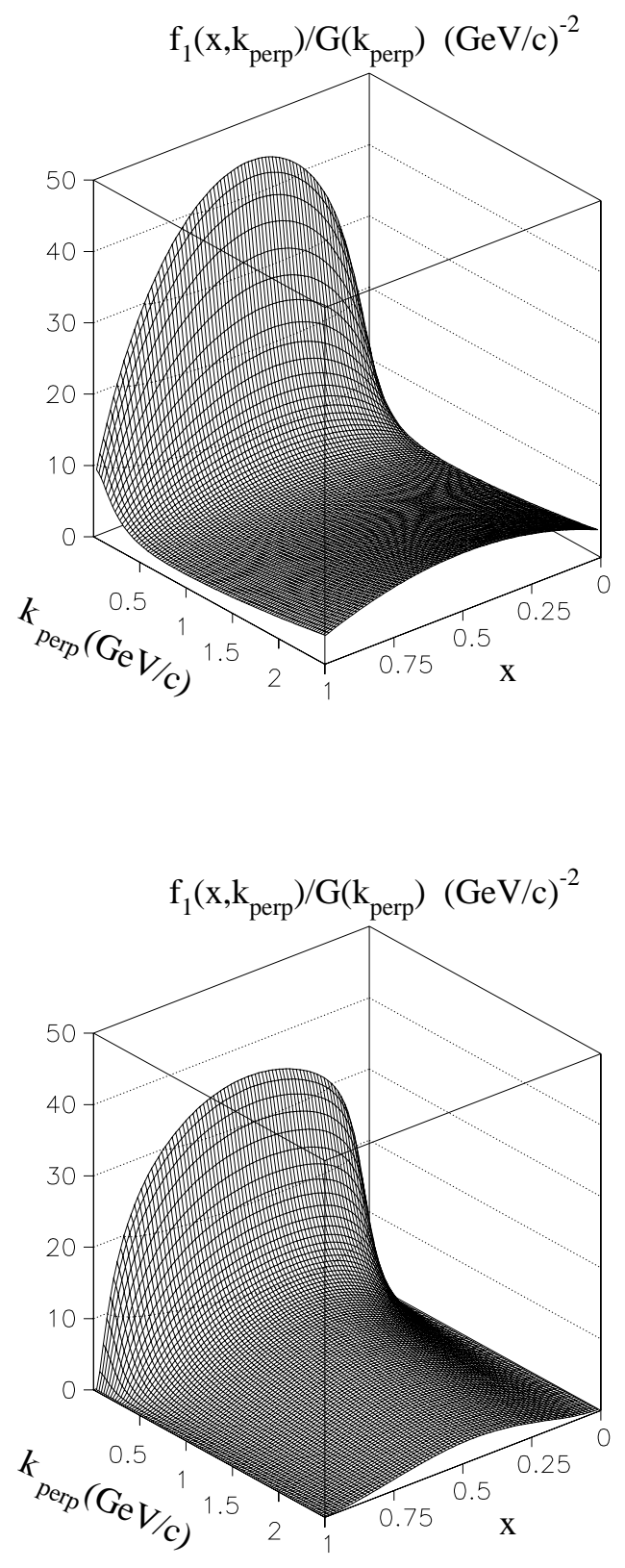

Fig. 5. Transverse-momentum dependent function, $f_{1}\left(x, k_{\perp}\right) / G\left(k_{\perp}\right)$, with $G\left(k_{\perp}\right)=1 /\left(1+\left|\mathbf{k}_{\perp}\right|^{2} / m_{\rho}^{2}\right)^{4}$. Upper panel: sum-form BSA. Lower panel: product-form BSA. The normalization is given by $\int_{0}^{1} d x \int d \mathbf{k}_{\perp} f_{1}\left(x, k_{\perp}\right)=1$ and $\mathrm{k}_{\text {perp }}=k_{\perp}$ (after Ref. [4]).

could argue that the product-form model contains the main ingredients for the description of the constituents inside the pion and could be applied to study experimental data. 

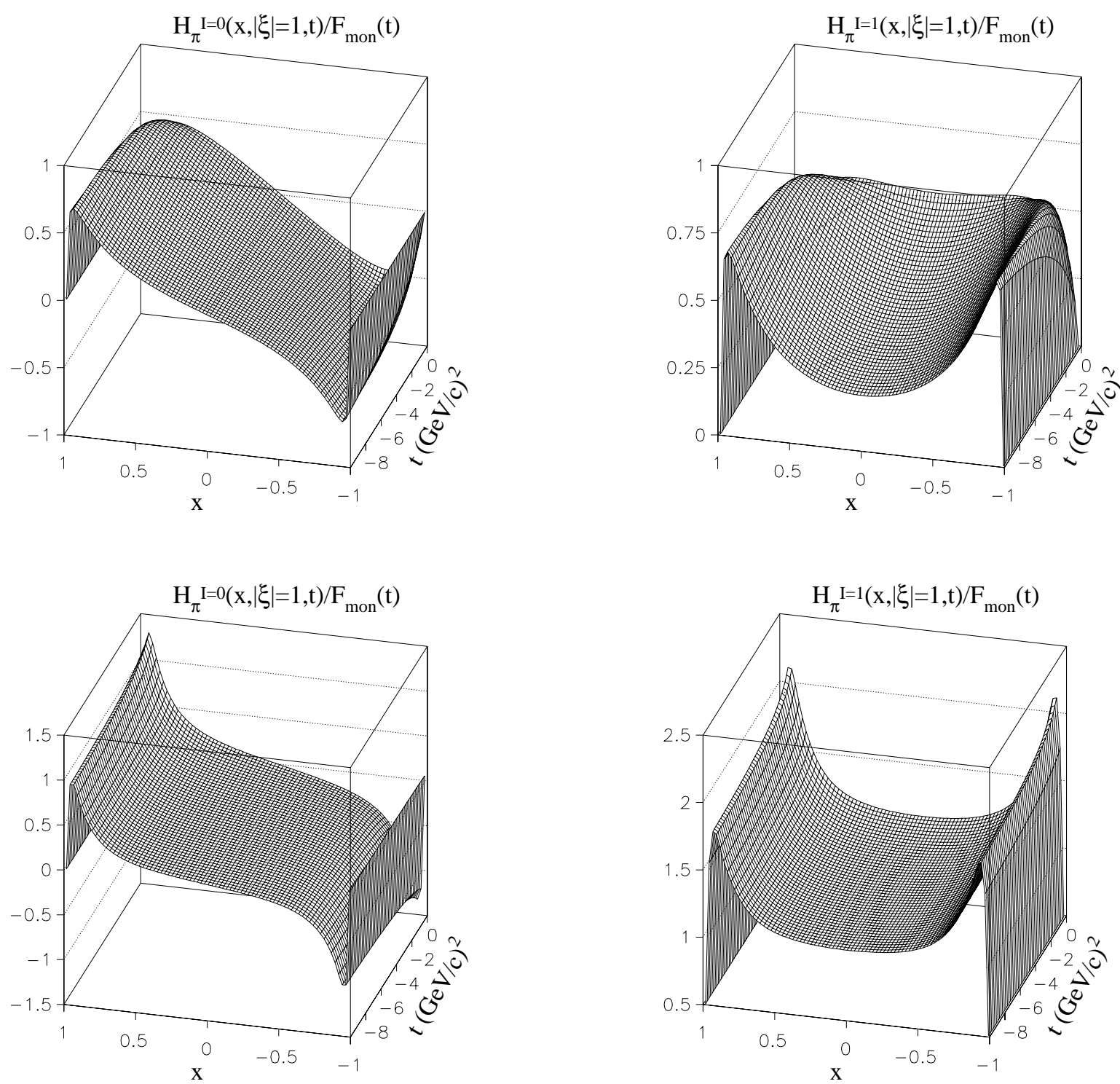

Fig. 6. Upper left panel: Isoscalar no-helicity flip GPD from the covariant symmetric model (product-form BSA) of Sec. 3.1 at $|\xi|=1$ (see text). Upper right panel: the same as in the left lanel, but for the isovector GPD. Lower panels: the same as in the upper panel, but for the Mandelstam-inspired model of Sec. 3.2 (after Ref. [4]).

\section{Nucleon relativistic model and nucleon parton momentum distributions}

We describe the quark-nucleon vertex function through a BSA, whose Dirac structure is suggested by an effective Lagrangian [19]. Then the symmetrized BSA for the nucleon is approximated as follows

$$
\begin{aligned}
\Phi_{N}^{\sigma}\left(k_{1}, k_{2}, k_{3}, P_{N}\right) & =\imath\left[S\left(k_{1}\right) \tau_{y} \gamma^{5} S_{C}\left(k_{2}\right) C \otimes S\left(k_{3}\right)\right. \\
& +S\left(k_{3}\right) \tau_{y} \gamma^{5} S_{C}\left(k_{1}\right) C \otimes S\left(k_{2}\right) \\
& \left.+S\left(k_{3}\right) \tau_{y} \gamma^{5} S_{C}\left(k_{2}\right) C \otimes S\left(k_{1}\right)\right]
\end{aligned}
$$

$$
\times \Lambda\left(k_{1}, k_{2}, k_{3}\right) \chi_{\tau_{N}} U_{N}\left(P_{N}, \sigma\right),
$$

where $S(k)$ is the CQ free propagator, $S_{C}(k)$ the charge conjugated propagator, $\Lambda\left(k_{1}, k_{2}, k_{3}\right)$ describes the symmetric momentum dependence of the vertex function upon the quark momentum variables, $k_{i}$, while $U_{N}\left(P_{N}, \sigma\right)$ is the nucleon spinor and $\chi_{\tau_{N}}$ the isospin eigenstate.

The matrix elements of the macroscopic current in the SL region are approximated microscopically by the Mandelstam formula [12]

$$
\left\langle\sigma^{\prime}, P_{N}^{\prime}\left|j^{\mu}\right| P_{N}, \sigma\right\rangle=3 N_{c}
$$



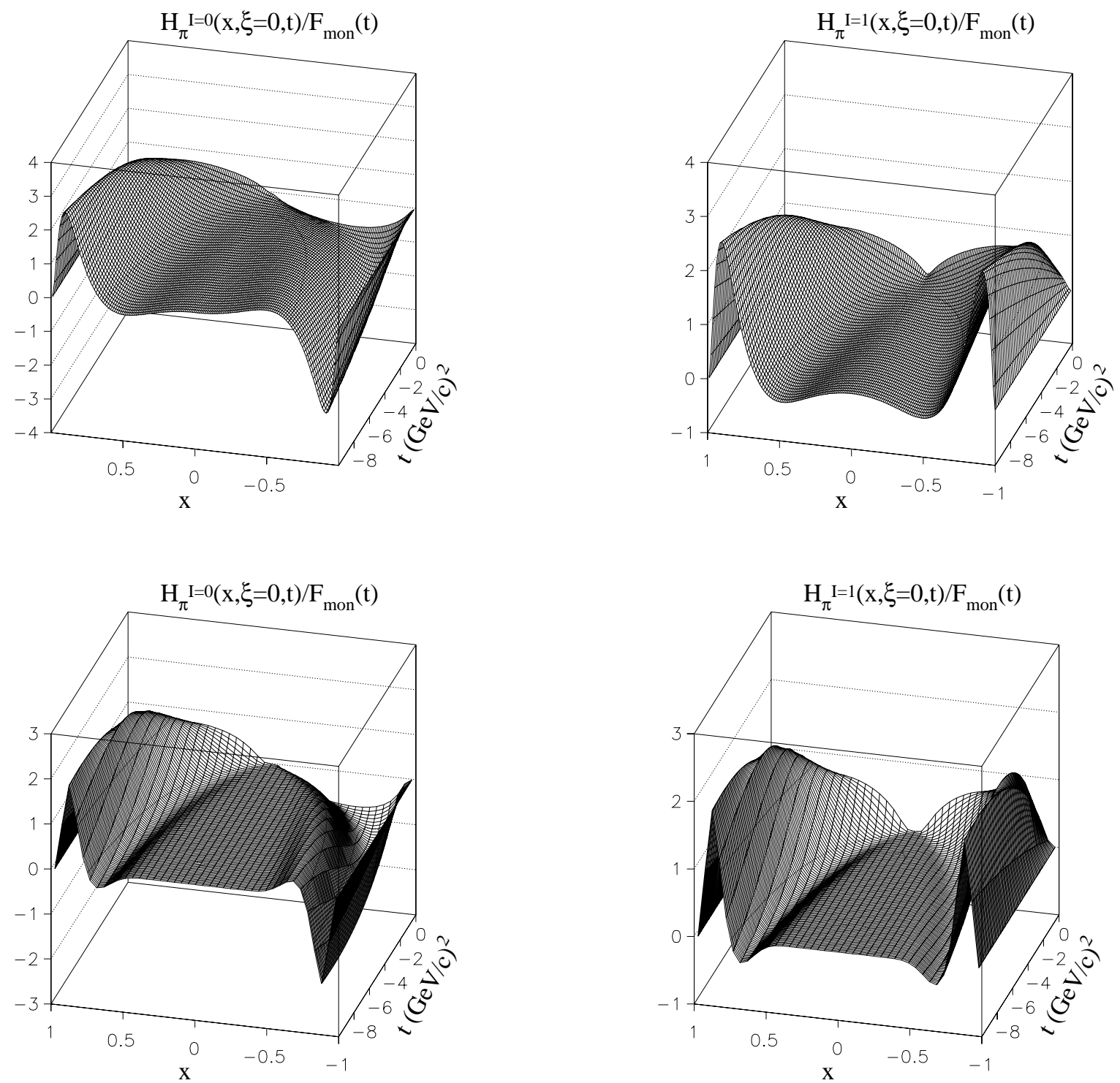

Fig. 7. Upper left panel: Isoscalar no-helicity flip GPD from the covariant symmetric model (product-form BSA) at $|\xi|=0$. Upper right panel: the same as in the left lanel, but for the isovector GPD. Lower panels: the same as in the upper panel, but for the LFHD model of Sec. 3.3 (after Ref. [4]).

$$
\begin{aligned}
& \times \int \frac{d^{4} k_{1}}{(2 \pi)^{4}} \int \frac{d^{4} k_{2}}{(2 \pi)^{4}} \sum\left\{\bar{\Phi}_{N}^{\sigma^{\prime}}\left(k_{1}, k_{2}, k_{3}^{\prime}, P_{N}^{\prime}\right)\right. \\
& \left.\times S^{-1}\left(k_{1}\right) S^{-1}\left(k_{2}\right) \mathcal{I}^{\mu}\left(k_{3}, q\right) \Phi_{N}^{\sigma}\left(k_{1}, k_{2}, k_{3}, P_{N}\right)\right\}
\end{aligned}
$$

where $k_{3}^{\prime}=k_{3}+q, I^{\mu}\left(k_{3}, q\right)$ is the quark-photon vertex, and $\sum$ implies a sum over isospin and spinor indexes. An analogous expression holds in the timelike (TL) region.

We adopt a Breit reference frame where $\mathbf{q}_{\perp}=0$ and $q^{+}=\left|q^{2}\right|^{1 / 2}$. Our CQ mass is $m=m_{u}=m_{d}=200 \mathrm{MeV}$.

The Mandelstam formula is projected out by an analytic integration on $k_{1}^{-}$and $k_{2}^{-}$, taking into account only the poles of the propagators. Then the current becomes the sum of a purely valence contribution (diagram (a) in Fig. 9) and a NV, pair-production contribution (diagram (b) in Fig. 9). Clearly, after the $k^{-}$integrations, the vertex functions depend only upon the LF three-momentum.

The quark-photon vertex has isoscalar (IS) and isovector (IV) contributions

$$
I^{\mu}=I_{I S}^{\mu}+\tau_{z} I_{I V}^{\mu}
$$

and each term in Eq. (25) contains a purely valence contribution (in the SL region only) and a contribution corresponding to the pair production (Z-diagram). In turn the Zdiagram contribution can be decomposed in a bare term $+a$ 


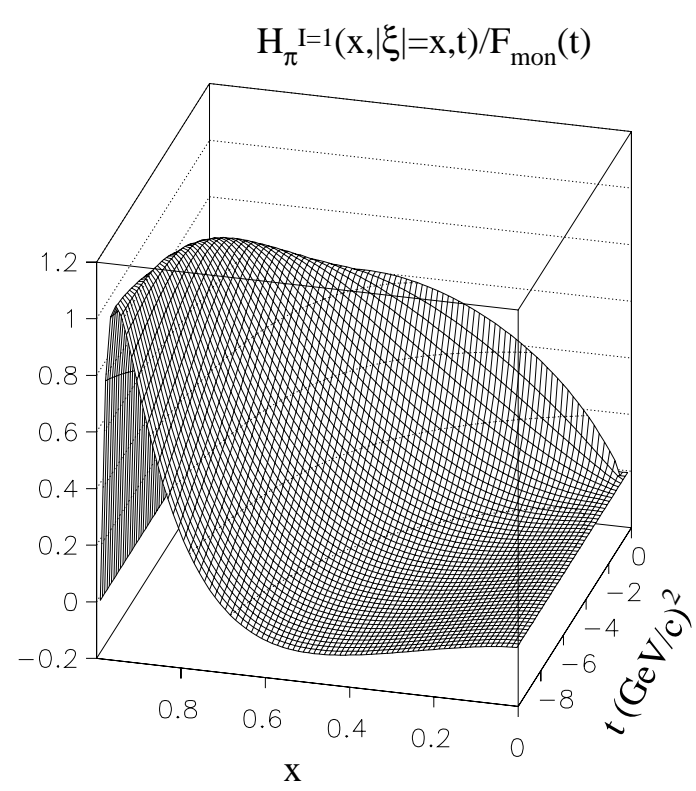

Fig. 8. Isovector unpolarized GPD at $|\xi|=x, m_{\pi}=0$ for the covariant model with the product form BSA (after Ref. [4]).

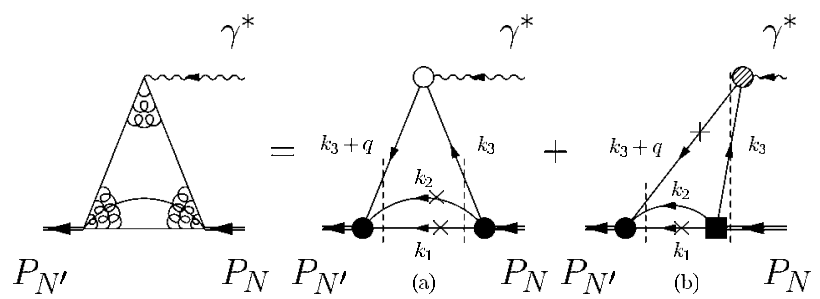

Fig. 9. Diagrams for the SL nucleon current: (a) valence (triangle) contribution with $0<k_{i}^{+}<P_{N}^{+}$, and $0 \leq k_{3}^{+}+q^{+}<P_{N}^{\prime+}$; (b) $\mathrm{NV}$, pair-production contribution with $0>k_{3}^{+} \geq-q^{+}$. A cross indicates a quark on the $k^{-}$-shell, i.e. $k^{-}=k_{o n}^{-}=\left(m^{2}+k_{\perp}^{2}\right) / k^{+}$. Solid circles and solid square represent valence and NV vertex functions, respectively (after Ref. [20]).

VMD term (according to the decomposition of the photon state in bare, hadronic [and leptonic] contributions), viz

$$
\begin{aligned}
& \mathcal{I}_{i}^{\mu}(k, q)=\mathcal{N}_{i} \theta\left(P_{N}^{+}-k^{+}\right) \theta\left(k^{+}\right) \gamma^{\mu}+\theta\left(q^{+}+k^{+}\right) \\
& \times \theta\left(-k^{+}\right)\left\{Z_{B} \mathcal{N}_{i} \gamma^{\mu}+Z_{V M}^{i} \Gamma^{\mu}(k, q, i)\right\}
\end{aligned}
$$

with $i=I S, I V, \mathcal{N}_{I S}=1 / 6$ and $\mathcal{N}_{I V}=1 / 2$. The constants $Z_{B}$ (bare term) and $Z_{V M}^{i}$ (VMD term) are unknown weights to be extracted from the phenomenological analysis of the experimental data.

According to the label $i$, the VMD term $\Gamma^{\mu}(k, q, i)$ includes isovector or isoscalar mesons. Indeed in [20] the microscopic model for the VMD, successfully used in [11] for the pion $\mathrm{ff}$ in the SL and in the TL region and based on the meson mass operator of Ref. [13], was extended to isoscalar mesons. As explained in [20], $\Gamma^{\mu}(k, q, i)$ does not involve free parameters. We consider up to 20 isoscalar and isovector mesons for achieving convergence at high $\left|q^{2}\right|$.

In the valence vertexes (solid circles in Fig. 9) the spectator quarks are on the $k^{-}$-shell, and the BSA momentum dependence is approximated through a nucleon wf a la Brodsky (PQCD inspired), namely

$$
\begin{aligned}
& \Psi_{N}\left(\tilde{k}_{1}, \tilde{k}_{2}, P_{N}\right)=P_{N}^{+} \frac{\left.\Lambda\left(k_{1}, k_{2}, k_{3}\right)\right|_{\left(k_{1 o n}^{-}, k_{2 o n}^{-}\right)}}{\left[m_{N}^{2}-M_{0}^{2}(1,2,3)\right]} \\
& \quad=P_{N}^{+} \mathcal{N} \frac{\left(9 m^{2}\right)^{7 / 2}}{\left(\xi_{1} \xi_{2} \xi_{3}\right)^{p}\left[\beta^{2}+M_{0}^{2}(1,2,3)\right]^{7 / 2}},
\end{aligned}
$$

where $\tilde{k}_{i} \equiv\left(k_{i}^{+}, \mathbf{k}_{i \perp}\right), M_{0}(1,2,3)$ is the free mass of the three-quark system, $\xi_{i}=k_{i}^{+} / P_{N}^{+}(i=1,2,3)$ and $\mathcal{N}$ a normalization constant.

The power $7 / 2$ and the parameter $p=0.13$ are chosen to have an asymptotic decrease of the triangle contribution faster than the dipole.

Only the triangle diagram determines the magnetic moments, which are weakly dependent on $p$. Then $\beta=0.645$ can be fixed by the magnetic moments and we obtain $\mu_{p}=$ $2.87 \pm 0.02\left(\mu_{p}^{\text {exp }}=2.793\right)$ and $\mu_{n}=-1.85 \pm 0.02\left(\mu_{n}^{\text {exp }}\right.$ $=-1.913)$.

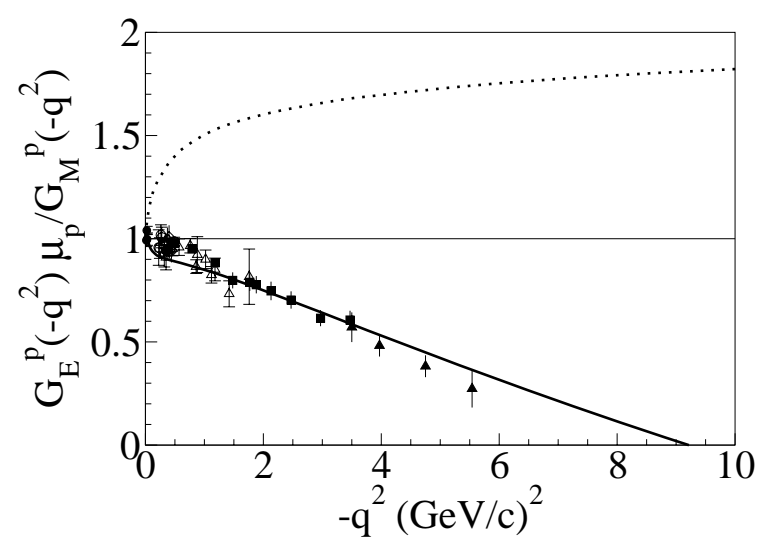

Fig. 10. Ratio between electric and magnetic ff's for the proton vs $-q^{2}$. Solid line: full calculation, sum of triangle and pairproduction terms; dotted line: triangle contribution only (after Ref. [20]).

For the Z-diagram contribution, the NV vertex (solid square in Fig. 9) is needed. It can depend on the available invariants, i.e. on the free mass, $M_{0}(1,2)$, of the $(1,2)$ quark pair and on the free mass, $M_{0}(N, \overline{3})$, of the ( nucleon - quark $\overline{3}$ ) system entering the NV vertex. Then in the SL region we approximate the momentum dependence of the NV ver$\operatorname{tex} \Lambda_{N V}^{S L}=\left.\Lambda\left(k_{1}, k_{2}, k_{3}\right)\right|_{k_{1 o n}^{-}, k_{3 o n}^{\prime}}$ by

$$
\Lambda_{N V}^{S L}=\left[g_{12}\right]^{2}\left[g_{N \overline{3}}\right]^{3 / 2}\left[\frac{k_{12}^{+}}{P_{N}^{\prime+}}\right]\left[\frac{P_{N}^{\prime+}}{k_{\overline{3}}^{+}}\right]^{r}\left[\frac{P_{N}^{+}}{k_{\overline{3}}^{+}}\right]^{r}
$$

with $k_{12}^{+}=k_{1}^{+}+k_{2}^{+}$and $g_{A B}=\left(m_{A} m_{B}\right) /\left[\beta^{2}+M_{0}^{2}(A, B)\right]$. The power 2 of $\left[g_{12}\right]^{2}$ is suggested from counting rules. The power $3 / 2$ of $\left[g_{N \overline{3}}\right]^{3 / 2}$ and the parameter $r=0.17$ are chosen to have an asymptotic dipole behavior for the NV contribution, as suggested by the OGE dominance.

Analogous expressions with the same parameters are used for the NV vertexes in the TL region (see Ref. [20] for the explicit expressions). 


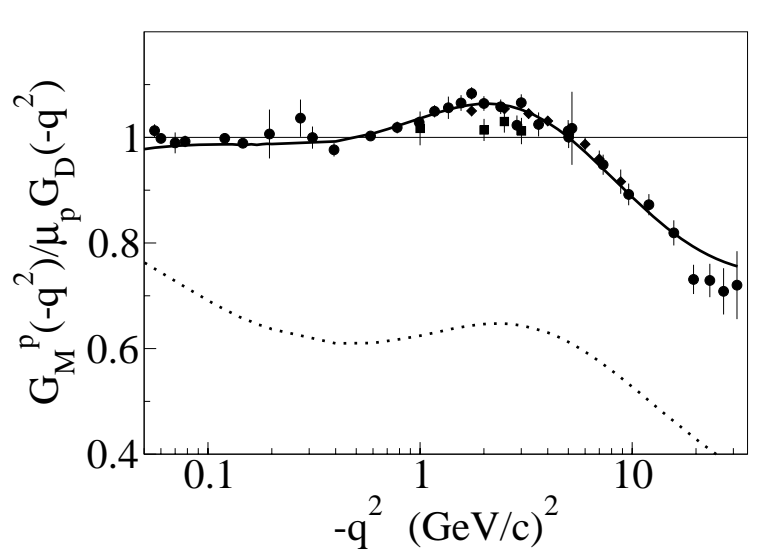

Fig. 11. Magnetic proton ff vs $-q^{2}$. Solid and dotted lines as in Fig. 10. $G_{D}\left(\left|q^{2}\right|\right)=\left[1+\left|q^{2}\right| /\left(0.71(\mathrm{GeV} / \mathrm{c})^{2}\right]^{-2}\right.$ (after Ref. [20]).

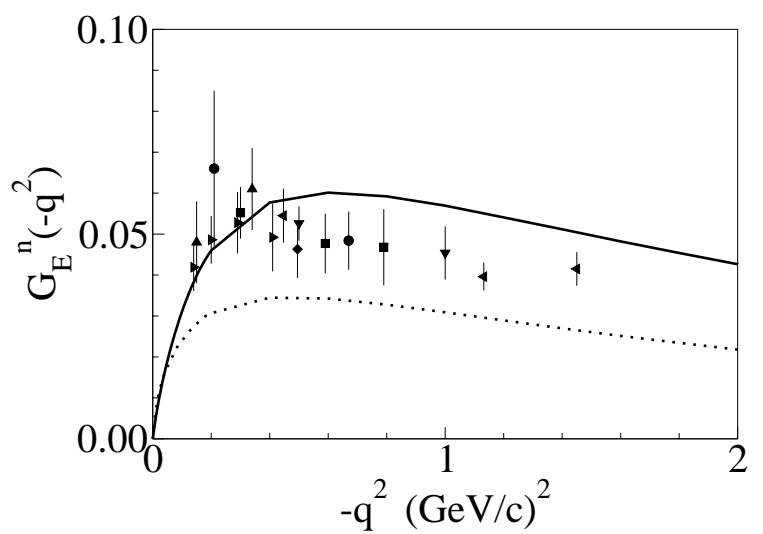

Fig. 12. Electric neutron $\mathrm{ff} v \mathrm{vs}-q^{2}$. Solid and dotted lines as in Fig. 10 (after Ref. [20]).

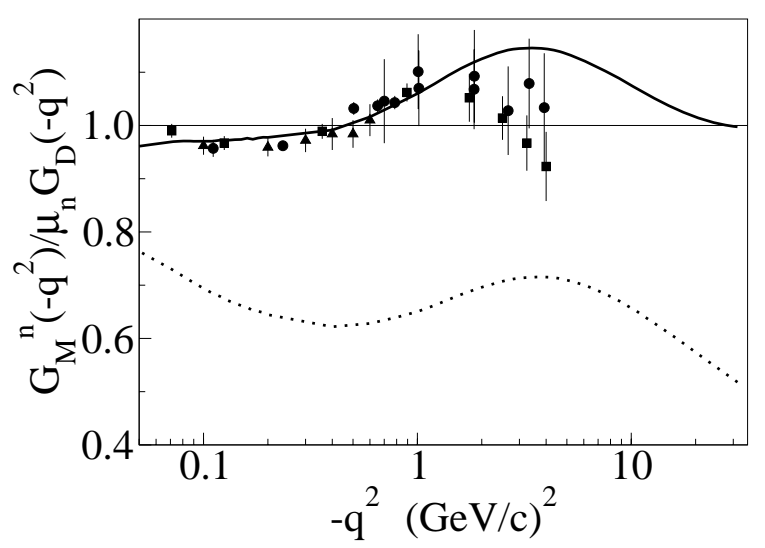

Fig. 13. The same as in Fig. 11, but for the neutron (after Ref. [20]).

\subsection{Nucleon form factors}

We perform a fit of our free parameters, $Z_{B}, Z_{V M}^{i}, p, r$ in the SL region and obtain the ff's shown in Figs. 10 13 , with a $\chi^{2} /$ datum $=1.7$. As a result the weights for the pair-production terms are $Z_{B}=Z_{V M}^{I V}=2.283$ and
$Z_{V M}^{I S} / Z_{V M}^{I V}=1.12$, remarkably close to one. The same values of our weight parameters are adopted to evaluate the TL ff's shown in Figs. 14 and 15.

Preliminary results of slightly different models for the nucleon ff's were presented in [21].

The Z-diagram turns out to be essential for the description of the ff's in our reference frame with $q^{+}>0$. In particular, the possible zero in $G_{E}^{p} / G_{M}^{p}$ for $q^{2}<0$ is strongly related to the pair-production contribution, i.e. to higher Fock state components.

In the TL region our parameter free calculations give a fair description of the proton data, apart for some missing strength at $q^{2}=4.5(\mathrm{GeV} / \mathrm{c})^{2}$ and $q^{2}=8(\mathrm{GeV} / \mathrm{c})^{2}$ (as occurs for the pion case [11]), which one could argue to be due to possible unknown vector mesons, missing in the spectrum of Ref. [13].

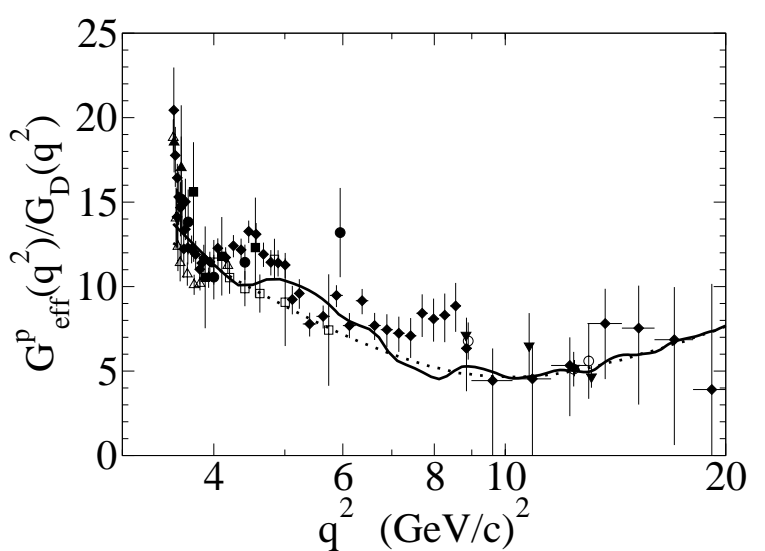

Fig. 14. Proton effective ff $G_{e f f}^{p}\left(q^{2}\right) / G_{D}\left(q^{2}\right)$ in the timelike region vs $q^{2}$. Solid line: bare term + VMD term; dotted line: bare term. $G_{e f f}\left(q^{2}\right)=\left[\left(\left|G_{M}\left(q^{2}\right)\right|^{2}-\eta\left|G_{E}\left(q^{2}\right)\right|^{2}\right) /(1-\eta)\right]^{0.5}$ with $\eta=$ $-2 m_{N}^{2} / q^{2}$ (after Ref. [20]).

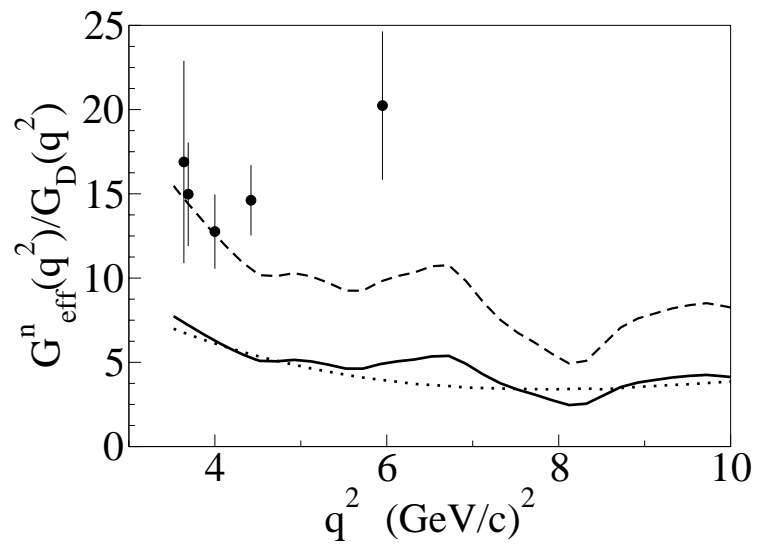

Fig. 15. The same as in Fig. 14, but for the neutron. Dashed line: solid line arbitrarily multiplied by 2 (after Ref. [20]). 


\subsection{Longitudinal and transverse quark momentum distribution in the nucleon}

The longitudinal distribution $q(x)$ is the limit in the forward case, $P_{N}^{\prime}=P_{N}$, of the unpolarized generalized parton distribution $H^{q}(x, \xi, t)$. Indeed, one can define the distributions $H^{q}(x, \xi, t)$ and $E^{q}(x, \xi, t)$ for the quark $q$ by

$$
\begin{aligned}
& \frac{1}{2 P^{+}} \bar{u}\left(P_{N}^{\prime}, \lambda^{\prime}\right)\left[H^{q} \gamma^{+}+E^{q} l \frac{\sigma^{+\alpha} q_{\alpha}}{2 M}\right] u\left(P_{N}, \lambda\right) \\
= & \left.\int \frac{d z^{-}}{4 \pi} e^{i x P^{+} z^{-}}\left\langle P_{N}^{\prime}, \lambda^{\prime}\left|\bar{\psi}_{q}\left(-\frac{z}{2}\right) \gamma^{+} \psi_{q}\left(\frac{z}{2}\right)\right| P_{N}, \lambda\right\rangle\right|_{\tilde{z}=0}
\end{aligned}
$$

where $\lambda$ is the nucleon helicity, while $P, x, \xi$, and $t$ have the same meaning as in Eqs. $(4,5)$, but with $p$ replaced by the nucleon momentum $P_{N}$, and $k$ is again the average momentum of the active quark, i.e. $k=\left[k_{3}+\left(k_{3}+q\right)\right] / 2$. For $P_{N}^{\prime}=P_{N}$, both $q^{+}$and $\xi$ are vanishing and $x=k_{3}^{+} / P_{N}^{+}=\xi_{3}$ coincides with the fraction of the longitudinal momentum carried by the active quark, i.e., with the Bjorken variable. As a consequence the function $H^{q}(x, \xi, t)$ reduces to the longitudinal parton distribution function $q(x)$ :

$$
\begin{gathered}
H^{q}(x, 0,0)=q(x)=\int d \mathbf{k}_{\perp} f_{1}^{q}\left(x, k_{\perp}\right) \\
=\left.\int \frac{d z^{-}}{4 \pi} e^{i x P^{+} z^{-}}\left\langle P_{N}\left|\bar{\psi}_{q}\left(-\frac{z}{2}\right) \gamma^{+} \psi_{q}\left(\frac{z}{2}\right)\right| P_{N}\right\rangle\right|_{\tilde{z}=0},
\end{gathered}
$$

where an average on the nucleon helicities is understood.

Once all the parameters of the nucleon light-front wf $\Psi_{N}\left(\tilde{k}_{1}, \tilde{k}_{2}, P_{N}\right)$ have been determined, one can easily define the TMD of the active quark in terms of the LF wf :

$$
\begin{aligned}
& f_{1}^{u(d)}\left(x, k_{\perp}\right)=\frac{N_{c}}{(2 \pi)^{6}} \frac{1}{P_{N}^{+2}} \\
& \times\left.\int_{0}^{1-x} d \xi_{2} \frac{1}{\left(1-x-\xi_{2}\right) \xi_{2}} \frac{1}{x^{2}} \int d \mathbf{k}_{2 \perp} \mathcal{H}_{u(d)}\right|_{\left(k_{1 o n}^{-}, k_{2 o n}^{-}\right)} \\
& \quad \times\left|\Psi_{N}\left(P_{N}^{+} \xi_{1}, \mathbf{k}_{1 \perp}, P_{N}^{+} \xi_{2}, \mathbf{k}_{2 \perp}, P_{N}\right)\right|^{2}
\end{aligned}
$$

where $\mathcal{H}_{u}$ and $\mathcal{H}_{d}$ are traces of propagators and of the currents $\mathcal{I}_{u}^{+}=\mathcal{I}_{I S}^{+}+\mathcal{I}_{I V}^{+}$and $\mathcal{I}_{d}^{+}=\mathcal{I}_{I S}^{+}-\mathcal{I}_{I V}^{+}$, respectively, with proper normalizations [5].

From the nucleon LF wf $\Psi_{N}\left(\tilde{k}_{1}, \tilde{k}_{2}, P_{N}\right)$ one can easily define through Eq. (30) also the longitudinal distribution of the struck quark and from the isospin symmetry one has

$$
u_{p}(x)=d_{n}(x)=u(x) ; \quad d_{p}(x)=u_{n}(x)=d(x)
$$

Our preliminary results for $f_{1}^{u(d)}\left(x, k_{\perp}\right)$ in the proton and for $u(x)$ and $d(x)$ are shown in Figs. 16, 17 and in Figs. 18,19 , respectively. It can be observed that the decay of our $f_{1}\left(x, k_{\perp}\right)$ vs $k_{\perp}$ is faster than in diquark models of the nucleon [22], while it is slower than in gaussian factorization models for the TMD [23].

As far as the longitudinal distributions are concerned, a reasonable agreement of our $u(x)$ with the CTEQ4 fit to the experimental data [24] can be seen in Fig. 18.

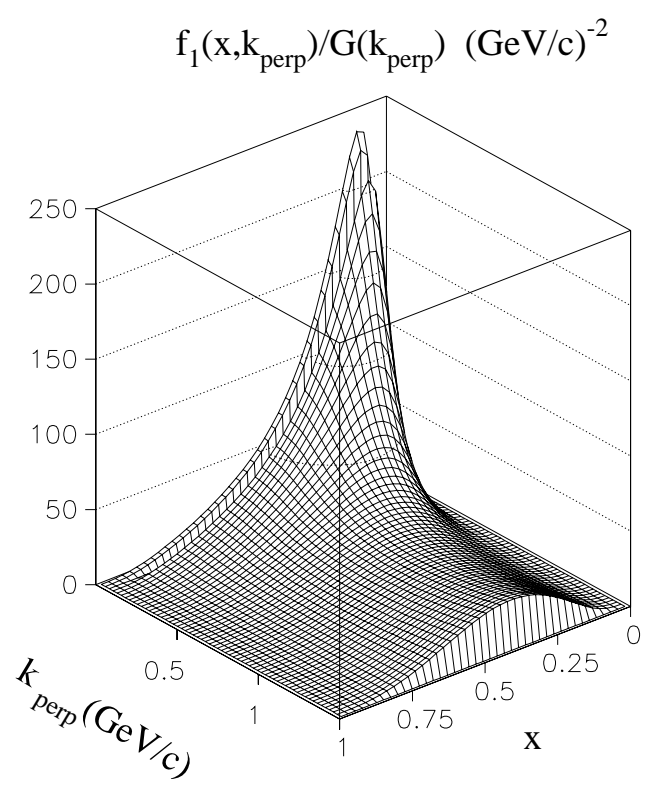

Fig. 16. Transverse-momentum distributions for a $u$ quark in the proton. $G\left(k_{\perp}\right)=\left(1+k_{\perp}^{2} / m_{\rho}^{2}\right)^{-5.5}, m_{\rho}=770 \mathrm{MeV}$ and $k_{\text {perp }}=k_{\perp}$ (after Ref. [5]).

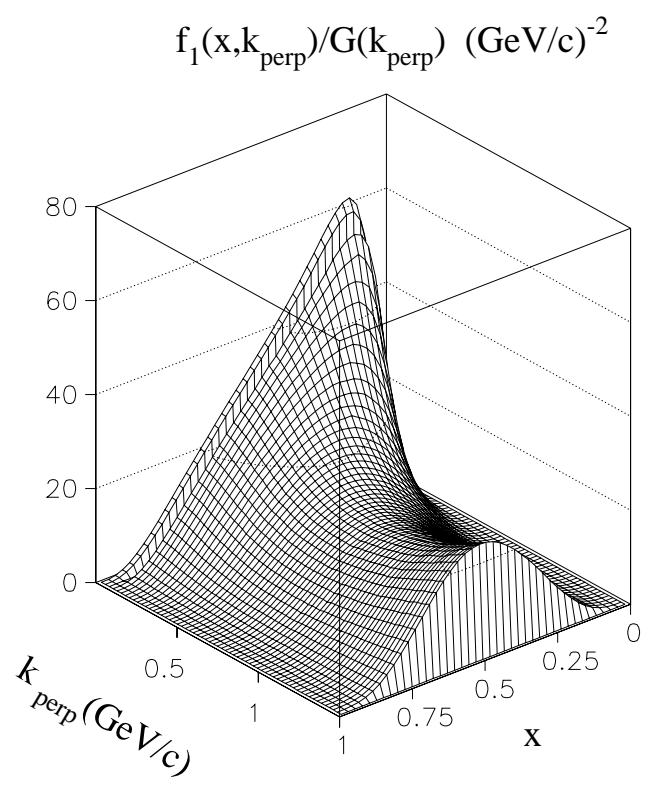

Fig. 17. The same as in Fig. 8, but for a $d$ quark inside the proton (after Ref. [5]).

\section{Conclusions}

Microscopical models for hadron em form factors have been investigated. Both for the pion and the nucleon good results are obtained in the SL region. The Z-diagram (i.e. higher Fock state component) has been shown to be essential, in reference frames where $q^{+} \neq 0$.

The analysis of the form factors allows us to get hadron vertexes that are used to evaluate the unpolarized longitudinal and transverse momentum distributions. The effects 


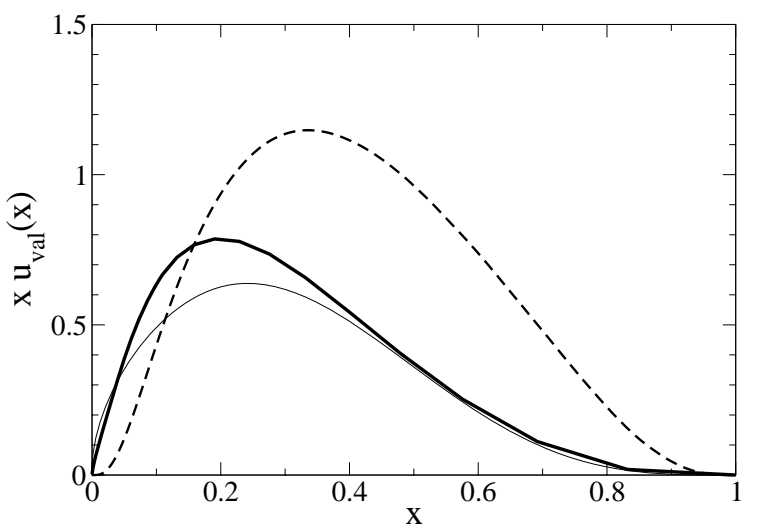

Fig. 18. Longitudinal momentum distribution for a $u$ quark inside the proton. Dashed lines: our preliminary results; thick solid lines: our results after evolution to $Q^{2}=1.6(\mathrm{GeV} / \mathrm{c})^{2}$; thin solid lines: CTEQ4 fit to the experimental data [24] (after Ref. [5]).

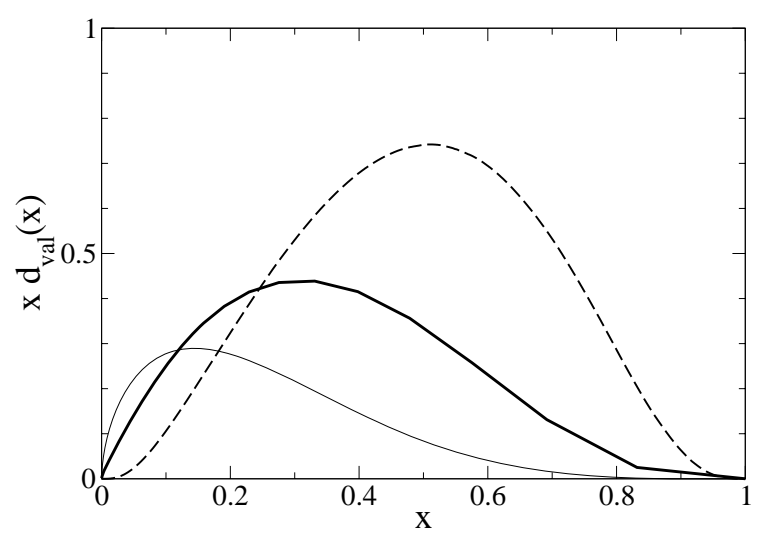

Fig. 19. The same as in Fig. 10, but for a $d$ quark in the proton (after Ref. [5]).

of a $k_{\perp}$ fall-off compatible with the OGE dominance has been explored. Its role for the description of the ff tail at high $-t$ and of vanishing longitudinal parton distributions at the end points has been shown.

Unpolarized pion GPD's are obtained within three different models. As $-t$ increases, the maximum of the GPD's migrates from $x \sim 0.5$ towards $x \sim 1$ and the kinematical origin of this behavior has been demonstrated.

Our next step will be the calculation of polarized transverse and longitudinal momentum distributions.

\section{References}

1. X.-D. Ji, J. Phys. G 24 (1998) 1181; Ann. Rev. Nucl. Part. Sci. 54, 413 (2004).

2. M. Diehl, Phys. Rep. 388 (2003) 41.

3. S. Boffi and B. Pasquini, Riv. Nuovo Cim. 30 (2007) 387.

4. T. Frederico, E. Pace, B. Pasquini, and G. Salmè, Phys. Rev. D 80 (2009) 054021.

5. E. Pace, J.P.B.C. de Melo, T. Frederico, S. Pisano and G. Salmè, to be published.

6. S.J. Brodsky, H.C. Pauli, and S.S. Pinsky, Phys. Rep. 301 (1998) 299.

7. V. N. Gribov, L. N. Lipatov, Sov. J. Nucl. Phys. 15 (1972) , 438 [Yad. Fiz. 15 (1972) 781]; 15 (1972) 675; G. Altarelli, G. Parisi, Nucl. Phys. B 126 (1977) 298; Yu. L. Dokshitzer, Sov. Phys. JETP 46 (1977) 641.

8. A. V. Efremov, A. V. Radyushkin, Phys. Lett. B 94 (1980) 245.

9. G. P. Lepage, S. J. Brodsky, Phys. Rev. D 22 (1980) 2157.

10. T. Frederico, G.A. Miller, Phys. Rev. D 45 (1992) 4207.

11. J.P.B.C. de Melo, T. Frederico, E. Pace and G. Salmè, Phys. Lett. B 581 (2004) 75; Phys. Rev. D 73 (2006) 074013.

12. S. Mandelstam, Proc. Royal Soc. A 233 (1956) 248.

13. T. Frederico, H.-C. Pauli and S.-G. Zhou, Phys. Rev. D 66 (2002) 116011.

14. C.-R. Ji and H.-M.Choi, Phys. Lett. B 513 (2001) 330.

15. P.L. Chung, F. Coester, and W.N. Polyzou, Phys. Lett. B 205 (1998) 545.

16. D. Brömmel et al., Eur. Phys. J. C 51 (2007) 335.

17. R. Baldini et al., Eur. Phys. J. C 11 (1999) 709; Nucl. Phys. A 666\&667 (2000) 38.

18. J. Volmer et al., Phys. Rev. Lett. B 86 (2001) 1713.

19. W.R.B. de Araujo, E.F. Suisso, T. Frederico, M. Beyer, H.J. Weber, Phys. Lett. B 478 (2000) 86; Nucl. Phys. A 694 (2001) 351.

20. J.P.B.C. de Melo, T. Frederico, E. Pace, S. Pisano and G. Salmè, Phys. Lett. B 671 (2009) 153.

21. E. Pace, G. Salmè, T. Frederico, S. Pisano and J.P.B.C. de Melo, Nucl. Phys. A 782 (2007) 69c; A 790 (2007) 606c.

22. R. Jacob, P.J. Mulders, J. Rodriguez, Nucl. Phys. A 626 (1997) 937.

23. M. Anselmino, E. Efremov, A Kotzinian, B. Parsamyan, Phys. Rev. D 74 (2006) 074015.

24. H.L. Lai et al., Phys. Rev. D 51 (1995) 4763.

\section{Acknowledgments}

This work was partially supported by the Brazilian agencies CNPq and FAPESP and by Ministero dell'Università e della Ricerca Scientifica e Tecnologica, as well as by HadronPhysics2, Grant Agreement No. 227431 under the Seventh Framework Program of the European Community. 